\title{
Quantum steering of electron wave function in an InAs $Y$-branch switch
}

\author{
G. M. Jones and C. H. Yang ${ }^{\text {a) }}$ \\ Department of Electrical and Computer Engineering, University of Maryland, College Park, \\ Maryland, 20742 \\ M. J. Yang \\ Naval Research Laboratory, Washington, DC 20375 \\ Y. B. Lyanda-Geller \\ Department of Physics, Purdue University, West Lafayette, Indiana 47907
}

(Received 12 July 2004; accepted 22 December 2004; published online 11 February 2005)

\begin{abstract}
We report experimental results on gated $Y$-branch switches made from InAs ballistic electron waveguides. We demonstrate that gating modifies the electron wave functions as well as their interference pattern, resulting in anticorrelated oscillatory transconductances. Our data provide evidence of steering the electron wave function in a multichannel transistor structure. (C) 2005 American Institute of Physics. [DOI: 10.1063/1.1867554]
\end{abstract}

Quantum effects in nanostructures provide insights into fundamental issues that cannot be addressed in atomic physics settings and offer perspectives for future applications in computing. ${ }^{1}$ In the regime where quantum effects dominate, electron transport exhibits different properties. For example, when the device size becomes less than the elastic mean-free path, electrons can traverse through the conductor ballistically, leading to conductance quantization. In addition, phase coherent transport plays an important role in nanometer-scale devices. Among devices exploiting these quantum effects, the $Y$-channel transistor is attractive on its own right. The original proposal of the $Y$-channel transistor, or $Y$-branch switch (YBS) (Ref. 2) came from an electron wave analogy to the fiber optic coupler. The semiconductor version of YBS has a narrow electron waveguide patterned into a " $Y$ " configuration with one source and two drain terminals. A lateral electric field perpendicular to the direction of electric current in the source waveguide steers the injected electron wave into either of the two outputs. YBS offers several advantages as a fast switch as evidenced by THz (Refs. 3 and 4) operation of quantum point contacts (QPC). Most interestingly, in the case of single mode occupation, the switching can be accomplished by a voltage of the order of $\hbar\left(e \tau_{t}\right)$, where $\tau_{t}$ is the transit time of electrons. ${ }^{5}$ With a proper design, the switching voltage for a YBS can become smaller than the thermal voltage, $k_{B} T / e$, as opposed to 40-80 times of $k_{B} T / e$ needed for the current transistors. Here, $k_{B}$ is the Boltzmann constant and $T$ is the absolute temperature. Switching at low voltages would make such devices less noisy and consume less power, though at the expense of speed. ${ }^{6}$

However, the experimental results on YBS and similar devices reported to date have yet to demonstrate the quantum switching. In almost all of the efforts, such as those in a $T$-branch, $Y$-junction, ${ }^{8-10}$ and ballistic rectifiers, ${ }^{11,12}$ the electrical characterizations were carried out at a source-drain bias significantly larger than $k_{B} T / e$. As a result, the transport mechanisms deviate too much away from the (near) equilibrium condition, which deems it necessary to maintain the long coherence length. Therefore, the coherent transport plays no significant role in the characteristics of devices reported so far. Recently, Monte Carlo simulation on the

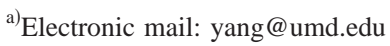

above-mentioned devices further indicates ${ }^{13}$ that all of the reported main features can be described classically without involving ballistic (and coherent) transport.

In this letter, we report an experimental demonstration of a YBS in the quantum regime using InAs electron waveguides. All transport characteristics reported here are acquired with an excitation voltage less than the thermal energy in order to keep the system near equilibrium. The characteristics of our YBSs show significant deviation from the classical transport features. Here, we will discuss the fabrication, transistor characteristics, and a qualitative explanation of the observed features.

Our transistors are built on InAs single-quantum wells (QWs) grown by molecular-beam epitaxy. A typical structure has a $2 \mu \mathrm{m}$ undoped AlSb buffer, a GaSb/AlSb smoothing superlattice, a $100 \mathrm{~nm}$ AlSb bottom barrier, and a $17 \mathrm{~nm}$ InAs QW. Two-dimensional (2D) electron gas in InAs QWs can have a long mean-free path, $\ell_{e}{ }^{14}$ and long coherence length. The surface Fermi-level pinning position in InAs is above its conduction-band minimum, allowing for the physical width of InAs conducting wires being only nanometers. ${ }^{15}$

Magnetotransport studies are used first to calibrate the $2 \mathrm{D}$ electron concentration $\left(n_{2 \mathrm{D}}\right)$ and mobility $\left(\mu_{2 \mathrm{D}}\right)$ of the as-grown sample. Quantum Hall plateaus and Shubnikov de Haas oscillations, both characterizing 2D electrons, are clearly observed on photolithographically patterned Hall bars. We obtained an $n_{2 \mathrm{D}}$ of $3.09 \times 10^{12} \mathrm{~cm}^{-2}, 2.08$ $\times 10^{12} \mathrm{~cm}^{-2}$, and $1.08 \times 10^{12} \mathrm{~cm}^{-2}$, and a $\mu_{2 \mathrm{D}}$ of 1.06 $\times 10^{4} \mathrm{~cm}^{2} / \mathrm{V} \mathrm{s}, \quad 1.67 \times 10^{4} \mathrm{~cm}^{2} / \mathrm{V} \mathrm{s}, \quad$ and $\quad 1.54$ $\times 10^{4} \mathrm{~cm}^{2} / \mathrm{V} \mathrm{s}$, at $300 \mathrm{~K}, 77 \mathrm{~K}$, and $4 \mathrm{~K}$, respectively. The corresponding Fermi wavelength, Fermi energy, and $\ell_{e}$ are calculated to be $14 \mathrm{~nm}, 140 \mathrm{meV}$, and $307 \mathrm{~nm}$ at $300 \mathrm{~K}, 17$ $\mathrm{nm}, 114 \mathrm{meV}$, and $397 \mathrm{~nm}$ at $77 \mathrm{~K}$, and $24 \mathrm{~nm}, 85 \mathrm{meV}$, and $264 \mathrm{~nm}$ at $4 \mathrm{~K}$, respectively. To verify the relatively long elastic mean-free path independently, we have used electronbeam lithography and wet etching to fabricate cross junctions for bend resistance measurements. ${ }^{16}$ At room temperature, $100 \mathrm{~nm}$ cross junctions indeed display negative bend resistance, ${ }^{17}$ which can be destroyed by magnetic focusing in a perpendicular magnetic field, further confirming the ballistic transport. ${ }^{18}$

The operation of YBS requires lateral electric field. We used an in-plane gate structure, ${ }^{19}$ in which two isolated, co- 

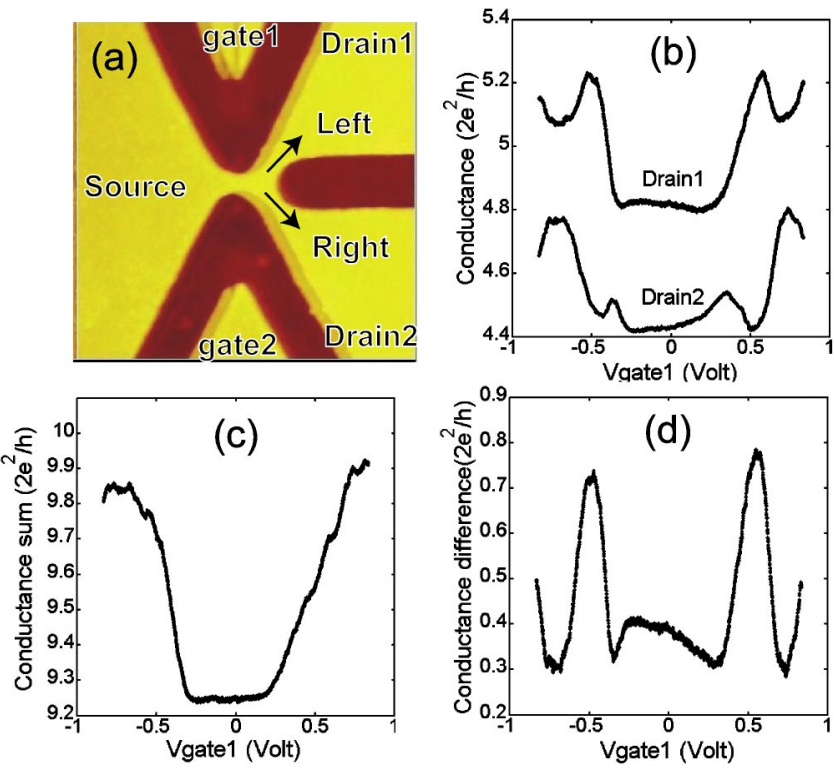

FIG. 1. (a) Atomic force micrograph of a finished YBS, where the narrowest neck has a width of $76 \mathrm{~nm}$. The dark region is etched and the AlSb buffer is revealed. InAs QW remains intact in the light region. The terminals for electrical measurement are labeled. (b) Measured conductances from Drain1 and Drain2 as a function of the differential gate voltage. (c) The sum of the two conductances. (d) Conductance of Drain1 is subtracted by that of Drain2.

planar, conductive regions on the opposite sides of the $Y$ junction are used as the two side gates. When we apply bias to these two side gates differentially, a lateral electric field perpendicular to the symmetry axis of the $Y$-junction arises. In contrast, with the two side gates shorted, a gate bias induces or depletes the electrons in the channel in the same way as a typical field-effect transistor does through capacitive coupling. The practical difficulty in realizing YBS has been in fabricating nanometer-scale electron waveguides without depleting electrons in narrow channels. We have overcome this hurdle by using InAs, for it has zero-lateral depletion width. The fabrication procedures are: First, the large-area bonding pads with leads are defined by photolithography, metal evaporation, and lift off. Second, electronbeam lithography is used to define the YBS patterns, as well as the two side gates. Third, using the patterned electronbeam resist as an etch mask, wet chemical etching is subsequently applied to form a trench, which physically and electrically isolates the side gates from the conductive $Y$ branches. Figure 1(a) shows an atomic force micrograph of a YBS device.

In order to verify the gating effect and to obtain insight into our sample system, we first characterized the QPC devices using the standard lock-in technique at $4.2 \mathrm{~K}$. The two side gates are shorted for a common-mode measurement. In the common-source configuration, the ac excitation voltage at the drain is kept low at $30 \mu \mathrm{V}_{\text {rms }}$ to avoid the undesirable, distracting "self-gating" effect. ${ }^{20}$ The drain current is fed to a transimpedance amplifier with an input impedance less than $10 \Omega$ and a gain of $1 \mathrm{M} \Omega$ or $10 \mathrm{M} \Omega$. The ac conductance is recorded as the gate voltage is swept from $-1 \mathrm{~V}$ to $1 \mathrm{~V}$. Figure 2 shows the typical current-voltage characteristics of a QPC and its numerical derivative. As expected, a series of plateaus, amplified as peaks in the derivative, is observed. The measured conductance may be expressed as $G_{N}$ $=1 /\left[1 /\left(N \cdot 2 e^{2} / h\right)+R_{S}\right]$, where $N$ is the number of one-

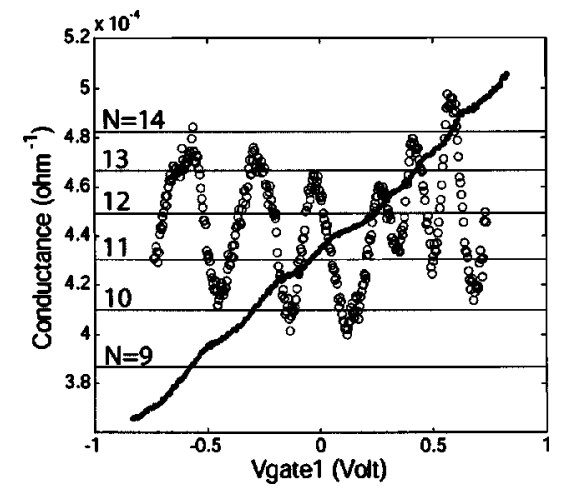

FIG. 2. The measured two-terminal source-drain conductance of a QPC and its numerical derivative (circles) as a function of common-mode gate biasing. The numbering of quantized conductance plateaus is also indicated. Within the shown gate bias range, there is no measurable dc gate leakage current $(\ll 1 \mathrm{pA})$.

dimensional spin degenerate channels and $R_{S}$ is series resistance. As shown by horizontal lines in Fig. 2 with $R_{S}$ $=1150 \Omega$, the calculated $G_{N}$ fits well with the measured data, and is in excellent agreement with the peak position in the derivative. This observation of the quantized conductance not only verifies the gating effect, but also demonstrates that the fabricated devices, including the QPCs and the YBSs, are indeed smaller than $\ell_{e}$.

Because of the ballistic coherent transport, the characteristics of gated YBS are expected to be drastically different from their classical counterparts, such as differential pair amplifiers. Figure 1(b) shows the transfer characteristics of our YBS with a $76 \mathrm{~nm}$ wide junction. There is no measurable gate leakage current $(\ll 1 \mathrm{pA})$ in our measurements. The transconductances through Drain1 and Drain2 are shown as a function of the sweeping differential gate voltage: $-0.83 \mathrm{~V}$ $<V_{\text {gate } 1}<0.83 V$, with $V_{\text {gate1 }}=-V_{\text {gate2. }}$. When $V_{\text {gate1 }}$ and $V_{\text {gate2 }}$ are swept, the electric field in the lateral direction steers the wave functions and the interference pattern of the injected electrons. Under such differential gating, the conductances through Drain1 and Drain2 show peaks and valleys, and these oscillatory features are anticorrelated. The sum and the difference of the two measured conductances are shown in Figs. 1(c) and 1(d), respectively.

The oscillatory transconductance is a manifestation of phase coherence in a multimode one-dimensional electron waveguide. Using a model InAs wire structure with $17 \mathrm{~nm}$ thick and $76 \mathrm{~nm}$ wide, we estimate that there are nine onedimensional modes populated under equilibrium at $4.2 \mathrm{~K}$. Conductance through Drain1 in our device is slightly bigger than that through Drain2, as shown in Fig. 1(b). Such asymmetry is a nonuniversal feature, resulting from variation in confinement potential, impurity distribution, surface roughness, and contacts in the device. In other words, the overall potential profile in the device defines the oscillatory features in conductance and the scale of these features is a fraction of $2 e^{2} / h$. This type of mesoscopic behavior is no surprise for devices with sizes smaller than the phase coherence length. ${ }^{21}$

We now qualitatively explain the feature in Fig. 1(c); the sum of two conductances increases with an increasing lateral electric field in either polarity. The eigenenergies of the transverse one-dimensional modes, the corresponding wave functions, and the longitudinal component of the electron wave functions are defined by the given device potential. Electrons in the $N$ th transverse channel propagating in a to AlP license or copyright, see http://apl.aip.org/apl/copyright.jsp 
waveguide from the source can be reflected back with amplitude $r_{N}$, transferred to the Drain1 waveguide with amplitude $\epsilon_{1 N}$ and transferred to the Drain2 waveguide with amplitude $\epsilon_{2 N}$. In a $Y$-switch with symmetric branches and symmetric confinement with respect to the axis of the source waveguide, odd number transverse modes have a maximum of the wave function on this axis. When scattering between transverse modes is ignored, the sum of conductances is given by $G_{\Sigma}=\Sigma_{N}\left(2 e^{2} / h\right)\left(\epsilon_{1 N}^{2}+\epsilon_{2 N}^{2}\right)=\Sigma_{N}\left(2 e^{2} / h\right)\left(1-r_{N}^{2}\right)$. When there is no transverse electric field, the reflection amplitude for an odd-numbered channel is maximal because electrons are strongly scattered by a beam splitter that separates Drain1 from Drain2. The corresponding contribution of odd-numbered channels to conductance is at its minimal. However, when a lateral electric field is applied, electrons are more likely to propagate into either Drain1 or Drain2, and are less scattered by the splitter. Therefore, reflection becomes weaker, and the sum of conductances through Drain1 and Drain2 becomes bigger. For even-numbered modes, the scattering off the beam splitter would likely increase with electric field, the reflection amplitude will grow, and the corresponding contribution to conductance decreases. However, because in our structure the total number of modes is odd (9), the gating effect in the odd channels prevails, and the sum of conductances through the two drains increases by a fraction of $2 e^{2} / h$. Another possible explanation of the observed behavior of $G_{\Sigma}$ is the increase of the number of conducting channels contributing to the current by one in the presence of transverse electric field. If the lateral confinement potential is parabolic, the energies of all transverse states decrease with applied electric field, resulting in more transverse states below the Fermi energy and an increased conductance. However, on the other hand, for rectangular confinement, the quantization energy of the ground state decreases, but energies of all other levels increase, and no addition of channels is allowed. This underscores the role of mesoscopic phase coherent effects and importance of specific realization of confinement (and impurity potential) for a given structure.

The observation that conductances through Drain1 and Drain2 are predominantly out of phase agrees well with the notion that the electric field steers the wave functions from one drain into the other. The individual Drain1 and Drain2 conductance characteristics are determined by the resonant transmission through quasi-localized states formed around the beam splitter. In conclusion, our observations provide strong evidence that the electron wave packet is being steered by an external electric field. The switching mechanism is quantum mechanical. Although the gate is capacitively coupled to the YBS as in classical transistors, the modulation of the drain current is through steering the wave functions. We have observed oscillatory transconductances that are always out of phase in the presence of multiple modes. ${ }^{22}$ Our observation not only verifies the quantum steering of electron wave functions in a semiconductor waveguide, but it also opens up possibilities for further studies of quantum switches in multiple-terminal nanometer-scale structure for information processing.

This work is supported in part by LPS and ONR. One of the authors (C.H.Y.) acknowledges discussion with Prof. P. T. Ho at the University of Maryland, and Drs. L. Thylén and E. Forsberg at the Royal Institute of Technology.

${ }^{1}$ Road Map to Nanoelectronics, edited by R. Compano, L. Molenkamp, and D. J. Paul 〈http://public.itrs.net/Files/2003ITRS/LinkedFiles/ERD/ NanoeletronicsRdmp.pdf $\rangle$

${ }^{2}$ T. Palm and L. Thylén, Appl. Phys. Lett. 60, 237 (1992).

${ }^{3}$ T. J. B. Janssen, J. C. Maan, J. Singleton, N. K. Patel, M. Pepper, J. E. F. Frost, D. A. Ritchie, and G. A. C. Jones, J. Phys.: Condens. Matter 6, L163 (1994)

${ }^{4}$ Q. Hu, S. Verghese, R. A. Wyss, T. Schapers, J. del Alamo, S. Feng, K. Yakubo, M. J. Rooks, M. R. Melloch, and A. Forster, Semicond. Sci. Technol. 11, 1888 (1996).

${ }^{5}$ T. Palm, L. Thylen, O. Nilsson, and C. Svensson, J. Appl. Phys. 74, 687 (1993).

${ }^{6}$ E. Forsberg, J. Appl. Phys. 93, 5687 (2003).

${ }^{7}$ I. Shorubalko, H. Q. Xu, I. Maximov, P. Omling, L. Samuelson, and W. Seifert, Appl. Phys. Lett. 79, 1384 (2001).

${ }^{8}$ L. Worschech, B. Weidner, S. Reitzenstein, and A. Forchel, Appl. Phys. Lett. 78, 3325 (2001).

${ }^{9}$ L. Worschech, H. Q. Xu, A. Forchel, and L. Samuelson, Appl. Phys. Lett. 79, 3287 (2001).

${ }^{10}$ K. Hieke and M. Ulfward, Phys. Rev. B 62, 16727 (2000).

${ }^{11}$ A. M. Song, A. Lorke, A. Kriele, J. P. Kothaus, W. Wegscheider, and M. Bichler, Phys. Rev. Lett. 80, 3831 (1998).

${ }^{12}$ A. M. Song, P. Omling, L. Samuelson, W. Seifert, I. Shorubalko, and H. Zirath, Jpn. J. Appl. Phys., Part 2 40, L909 (2001).

${ }^{13}$ J. Mateos, B. G. Vasallo, D. Pardo, T. González, J.-S. Galloo, S. Bollaert, Y. Roelens, and A. Cappy, IEEE Trans. Electron Devices 50, 1897 (2003).

${ }^{14}$ S. J. Koester, B. Brar, C. R. Bolognesi, E. J. Caine, A. Patlach, E. L. Hu, and H. Kroemer, Phys. Rev. B 53, 13063 (1996); C. H. Yang, M. J. Yang, K. A. Cheng, and J. C. Culbertson, ibid. 66, 115306 (2002).

${ }^{15}$ K. A. Cheng, C. H. Yang, and M. J. Yang, Appl. Phys. Lett. 77, 2861 (2000); J. Appl. Phys. 88, 5272 (2000); T. H. Chang, C. H Yang, and M. J. Yang, Phys. Status Solidi B 224, 693 (2001).

${ }^{16}$ G. Timp, H. U. Baranger, P. deVegvar, J. E. Cunningham, R. E. Howard, R. Behringer, and P. M. Mankiewich, Phys. Rev. Lett. 60, 2081 (1988).

${ }^{17}$ G. M. Jones, M. J. Yang, and C. H. Yang (unpublished).

${ }^{18}$ C. W. J. Beenakker and H. van Houten, Solid State Physics, edited by H. Ehrenreich and D. Turnbull (Academic, Boston 1991), Vol. 44.

${ }^{19}$ K. K. de Vries, P. Stelmaszyk, and A. D. Wieck, J. Appl. Phys. 79, 8087 (1996).

${ }^{20}$ J.-O. Wesstrom, Phys. Rev. Lett. 82, 2564 (1999); S. Reitzenstein, L. Worschech, P. Hartmann, M. Kamp, and A. Forchel, ibid. 89, 226804 (2002).

${ }^{21}$ Mesoscopic Phenomena in Solids, edited by B. L. Altshuler, P. A. Lee, and R. A. Webb (North-Holland, Amsterdam, New York, 1991).

${ }^{22}$ T. Palm, J. Appl. Phys. 74, 3551 (1993); Phys. Rev. B 52, 13773 (1995). This simulation of a multiple-mode YBS shows an in-phase oscillatory conductance versus a sweeping gate bias, and this feature is explained by mode occupation. In contrast, we observed out-of-phase oscillatory conductances in the presence of multiple modes. 